\title{
Irrungen und Wirrungen in der Erfassung der Myokardinfarkt-Mortalität in der Schweiz
}

\author{
Im DRG-System wird zu Abrechnungszwecken kodiert - daraus generierte Letalitäts- \\ daten sind zur Versorgungsqualitätsmessung nur bedingt tauglich oder gar untaug- \\ lich, denn unterschiedliche Bewertungen von diagnostischen Kriterien führen \\ unabdingbar zur Verzerrung von Quotienten, die als Letalitätsdaten die Qualität \\ eines Leistungserbringers reflektieren sollten.
}

Florim Cuculia,

Thomas Kaufmann ${ }^{b}$, Guido Schüpfer ${ }^{b}$

a Abteilung für Kardiologie, Department Innere Medizin Luzerner Kantonsspital, Luzern

b Medizinischer Stab, Qualitäts- und Risikomanagement, Luzerner Kantonsspital, Luzern

* Die Literatur findet sich unter www.saez.ch $\rightarrow$ Aktuelle Nummer oder $\rightarrow$ Archiv $\rightarrow$ $2014 \rightarrow 36$.

Korrespondenz:

Dr. med. Guido Schüpfer, PhD MBA HSG

Leiter Medizinischer Stab

Luzerner Kantonsspital

CH-6000 Luzern 16

Tel. 0412054301

guido.schuepfer[at]luks.ch

\section{Einführung}

Das Bundesamt für Gesundheit (BAG) publiziert auf seiner Website (www.bag.admin.ch) jährlich Qualitätsindikatoren für das Schweizer Gesundheitswesen. Die Myokardinfarkt-Krankenhausmortalität gehört zu den wichtigen Qualitätsindikatoren und hat in den letzten Jahren immer wieder Anlass zu Diskussionen in den Medien gegeben. Das Luzerner Kantonsspital (LUKS) hatte bereits im Jahr 2008 eine sehr hohe Myokardinfarkt-Mortalität von 12,3\% (bei einer laut BAG zu erwartenden Mortalität von 7,7\%). Das Medienecho darüber war entsprechend unerfreulich und für das engagierte Personal belastend und desavouierend. Diese Zahlen wurden in unserer Klinik hinterfragt, diskutiert und lösten die Überprüfung der Datenerhebung, aber auch von verschiedenen Behandlungsprozessen aus. Auch 2013 dürfte die Infarktletalitätsrate des LUKS mit 7,7\% im Vergleich zu anderen Leistungserbringern deutlich $\mathrm{zu}$ hoch sein und erneut zu diskutieren geben.

Nachfolgend werden daher die Problematik der auf Codierdaten basierenden Mortalitätsraten erläutert, Probleme bei der Diagnose-Stellung beim Myokardinfarkt diskutiert und Lösungsvorschläge für die Zukunft gemacht.

\section{Der ST-Hebungsinfarkt muss Top-Priorität im Katheterlabor haben}

Das Elektrokardiogramm ist immer noch das wichtigste Tool in der Diagnose-Stellung und Einleitung der Behandlung beim Myokardinfarkt. Es wird zwischen Myokardinfarkt mit ST-Hebungen (STEMI) [1] * und ohne ST-Hebungen (NSTEMI) [2] unterschieden und Patienten mit einem STEMI sollten so schnell wie möglich eine primäre perkutane Koronarintervention (PPCI) erhalten. Das Luzerner Kantonsspital in Luzern bildet mit den Spitälern der Region (Sursee, Wolhusen, Stans und Sarnen) ein MyokardinfarktNetzwerk und Patienten mit STEMI haben eine absolute Priorität im Katheterlabor. Das EKG wird vom

\section{Errements et tourments dans le} recensement de la mortalité liée à l’infarctus du myocarde en Suisse

Pour différentes raisons, les données sur la mortalité peuvent difficilement faire office d'indicateurs de qualité dans le but d'évaluer un fournisseur de prestations, et de ce fait, pour prévenir les cas de décès susceptibles d'être évités. Dans le système DRG, les données sont codées à des fins de facturation: les données sur la létalité qui sont générées par ce biais ne sont donc que partiellement voire pas du tout appropriées pour évaluer la qualité des soins, sachant que les différentes méthodes utilisées pour déterminer les critères diagnostiques mènent inévitablement à un biais des quotients (c'est-à-dire des données sur la létalité) censés refléter la qualité d'un prestataire. Pour obtenir des indicateurs plus fiables et objectifs, il est impératif d'établir des définitions claires mais également d'élargir le catalogue DRG afin de pouvoir notamment coder les patients qui sont référés aux hôpitaux en fin de vie. Une analyse de la qualité des processus et une comparaison des données avec celles d'un partenaire de benchmarking mettent en question l'adéquation des indicateurs de létalité en tant qu'indicateurs de qualité pour le traitement de l'infarctus du myocarde, à l'exemple de l'Hôpital cantonal de Lucerne. En effet, les données sur la qualité de l'indication et des processus nous semblent mieux à même de contribuer à améliorer la qualité des soins. 
Rettungsdienst beim Patienten geschrieben und über Smartphone direkt zum diensthabenden Kardiologen und zum Notfallmediziner übermittelt. Der Patient mit STEMI wird dann vom Rettungsdienst direkt ins Katheterlabor gebracht. Um sicherzustellen, dass der STEMI die notwendige Priorität erhält, wird seit fast 2 Jahren die Door-to-balloon-(DTB-)Zeit prospektiv erfasst, also die Zeit vom Eintreffen des Patienten bis zur Rekanalisation der verschlossenen Koronararterie. Die DTB-Zeit wird intern publiziert und diskutiert. Die mittlere Door-to-balloon-Zeit betrug im ersten Trimester 201426 Minuten (95\% CI: 17-34 Minuten), was deutlich unter der von der Europäischen Gesellschaft für Kardiologie (ESC) empfohlenen Zeit von 60 Minuten liegt [1].

\section{Der NSTEMI ist nicht einheitlich definiert}

Mortalitätsraten werden über einen Zähler und einen Nenner gebildet. Die Myokardinfarkt-Letalitätsrate eines Leistungserbringers ergibt sich aus den codierten verstorbenen Myokardinfarktpatienten, bezogen auf alle kodierten Infarkte. Damit die Rate akkurat ist, müssen daher sowohl Zähler und Nenner valide sein.

Während der STEMI ein relativ klar definiertes Krankheitsbild ist als auch auch eine nachvollziehbare Pathophysiologie hat (in den meisten Fällen: Plaqueruptur, gefolgt vom akuten Verschluss einer Koronararterie) werden im Begriff NSTEMI mehrere klinische Syndrome zusammengefasst [2]. Das Hauptkriterium für die Diagnose eines Myokardinfarkts ist ein dokumentierter Anstieg und Abfall von kardialen Biomarkern (bevorzugt Troponin), wobei mindestens ein Wert über der 99 Perzentile des oberen Referenz-Grenzwerts liegen muss [3]. Die ESC unterscheidet Typen von Myokardinfarkten, wobei Typ 1 (spontaner Myokardinfarkt) dem entspricht, was man schon immer unter dem Begriff «Myokardinfarkt» verstand, also meistens bedingt durch Plaqueruptur, gefolgt von Thrombose und verlangsamtem Koronafluss. Viel problematischer ist die Diagnose des Typ-2-Myokardinfarkts, dem ein Ungleichgewicht zwischen Angebot und Nachfrage an myokardialem Sauerstoff zugrunde liegt. Hierzu gehören ein Sammelsurium an klinischen Syndromen wie Koronarspasmus, Tachy- und Bradyarrhythmien, Anämie, respiratorische Insuffizienz, Hypooder Hypertonie usw. Die Unterscheidung zwischen Typ-1- und Typ-2-Myokardinfarkt ist im Alltag schwierig und bei vielen Patienten ohne die Durchführung einer Koronarangiographie praktisch unmöglich.

\section{Die Regulierungsbehörden meinen es gut, aber...}

Wir finden Transparenz im Gesundheitswesen grundsätzlich gut und unterstützen die Publikation der Mortalitätsstatistik durch das BAG. Die vom BAG pu- blizierten Zahlen sind aber aus mehreren Gründen zumindest problematisch.

Das BAG schreibt weder vor, wie die Diagnose des Myokardinfarkts zu stellen ist, noch werden die Daten diesbezüglich auditiert. Es macht einen grossen Unterschied, ob man den Myokardinfarkt aufgrund der Erhöhung der MB-Fraktion der CreatinKinase stellt oder ob man einen hochsensitiven Troponin-Assay verwendet. Durch den Gebrauch von hochsensitiven Troponin-Assays wird man mehr Myokardinfarkte diagnostizieren, aber dies ist klinisch nicht immer sinnvoll [4]. Will man also die Qualität der Behandlung des Myokardinfarkts vergleichen, dann muss als erstes sichergestellt werden, dass alle vom Gleichen reden, also auf einer klaren Definition fussen.

Als Beispiel kann man Patienten nehmen, die sich mit einer supraventrikulären Tachykardie (SVT) vorstellen [5]. Diese haben häufig eine minime Troponin-Erhöhung, die auch eine Dynamik zeigt. Zusammen mit den klinischen Symptomen (thorakales Engegefühl, Atemnot) sollte man hier von einem Myokardinfarkt reden. Die meisten Patienten mit SVT und leichter Troponin-Erhöhung haben keine KHK, die Diagnose eines Myokardinfarkts macht also klinisch wenig Sinn, sie entspricht jedoch den aktuellen Richtlinien [3]. Dazu kommt, dass diese Patienten meistens eine gute Prognose haben, da es sich tendenziell um jüngere Patienten handelt.

Die andere Seite der Medaille bilden Patienten auf der Intensivstation, die im Rahmen ihrer Krankheit (z. B. schwere Sepsis) eine Erhöhung des Troponins haben [6]. Auch hier sagt das Troponin nicht unbedingt etwas über das Ausmass der koronaren Herzkrankheit aus, aber es ist bekannt, dass diese Patienten eine schlechte Prognose haben (und zwar durch die Grundkrankheit und nicht durch den Myokardinfarkt).

Ein Spital könnte also theoretisch die Sterblichkeits-Rate über den Nenner steuern, indem es Myokardinfarkte bei Patienten mit SVT diagnostiziert, dies jedoch bei Patienten auf der Intensivstation unterlässt. Deshalb braucht es eine einheitliche Regelung, wie und bei wem ein Myokardinfarkt diagnostiziert werden kann, damit ein Vergleich zwischen den Spitälern sinnvoll ist.

\section{Die «Out-of-hospital»-Reanimierten - nicht nur ein ethisches Problem}

Patienten nach «Out-of-hospital»-Reanimation haben eine schlechte Prognose, und laut einer $2010 \mathrm{pu}$ blizierten Meta-Analyse schaffen es nur 7,6\% (95\% CI, 6,7-8,4), das Spital lebend zu verlassen [7]. In unserem Spital liegt es in der Entscheidungskompetenz des Rettungsdienstes, ob sie einen Patienten nach Herz-Kreislauf-Stillstand ohne spontanen Kreislauf ins Spital fahren oder nicht. Ermuntert man also den Rettungsdienst, Patienten ohne spontanen Kreislauf 
ins Spital zu fahren (und nimmt eine höhere Mortalitäts-Rate in Kauf), oder lässt man die Leute lieber ausserhalb des Spitals sterben und hat dafür eine tiefere Mortalitäts-Rate? Was es braucht, ist ein spezieller Code für solche Patienten, denn ob ein solcher Patient stirbt oder nicht, hat in den meisten Fällen nichts mit der Qualität der Reanimation oder der initialen Behandlung zu tun.

\section{Palliative Therapie bei älteren und/oder multimorbiden Patienten}

Folgt man der Logik, dass das Spital mit der tiefsten Mortalitätsrate das beste Spital ist, muss man alles tun, damit niemand im Spital stirbt. Der Tod bedeutet jedoch für gewisse Patienten eine Erlösung, und wir sind als Ärzte auch verpflichtet, den Wunsch der Patienten zu respektieren. Die Patientin mit dem

\section{«Lässt man die Leute lieber ausserhalb des Spitals sterben und hat dafür eine tiefere Mortalitäts-Rate?»}

metastasierten Pankreaskarzinom soll auch an einem Myokardinfarkt sterben dürfen.

\section{Problemstellung}

Die folgenden Auswertungen basieren auf den revidierten Kodierdatensätzen des Jahres 2013 des LUKS und eines Benchmark-Spitals. Ein zum LUKS absolut vergleichbares Spital hat sich als Benchmark zur Verfügung gestellt, möchte jedoch nicht namentlich erwähnt werden. Es wurde nach der ICD-10-Diagnose I.21.x gefiltert, d.h. alle Patienten, die in der BAG-Q-Indikatoren-Auswertung als «Myokardinfarkt» geführt werden.
Die Myokardinfarkt-Mortalitätsrate des LUKS betrug 2013 7,7\% während der Vergleich eine Rate von $5,1 \%$ aufwies. Trotz Anwendung von evidenzbasierter Therapie, Standard Operating Procedures, regelmässigen Morbiditäts- und Mortalitätskonferenzen und guten Door-to-balloon-Zeiten, ist die Gesamtletalitätsrate für einen Myokardinfarkt am LUKS hoch. Wir finden aktuell keine Hinweise auf insuffiziente Prozesse und, wie in Tabelle 1 dargestellt, unterscheidet sich unser Kollektiv auf der Intensivstation nicht vom Benchmark. Das Durchschnittsalter der IPS-Patienten lag im LUKS bei 65,9 Jahren im Vergleich zum Benchmarkpartner bei 63,2 Jahren. Ob es sich trotz gleicher Diagnose um ein vergleichbares Kollektiv handelt, lässt sich durch einfache Kennzahlen schätzen: neben dem Alter sind der patientenbezogene Schweregrad (PCCL) und das cost weight (CW) ein Mass für den Schweregrad der Erkrankung. Da es sich um grosse Populationen handelt, müssten die Werte mit dem Benchmarkpartner vergleichbar sein.

Am LUKS werden Patienten mit einem erhöhten Gesamtschweregrad behandelt (statistisch signifikant), was mit einer höheren Mortalität assoziiert ist. Ein Teil der erhöhten Mortalität kann also durch unterschiedliche Patientenkollektive erklärt werden.

Das Benchmark-Spital hat ein etwas kleineres Einzugsgebiet, hat aber im gleichen Zeitraum ca. 20\% mehr Myokardinfarkte kodiert. Auch die Zahl der invasiven Behandlungen (PTCA) ist im BenchmarkSpital deutlich kleiner, was vermuten lässt, dass es sich bei vielen Myokardinfarkten nicht um Typ-1-Infarkte handelt, wo eine Plaque-Ruptur ursächlich ist und somit eine invasive Behandlung am meisten bringt.

Da die vorgängigen Analysen keine Defizite in der Prozess- oder Strukturqualität vermuten lassen, ist

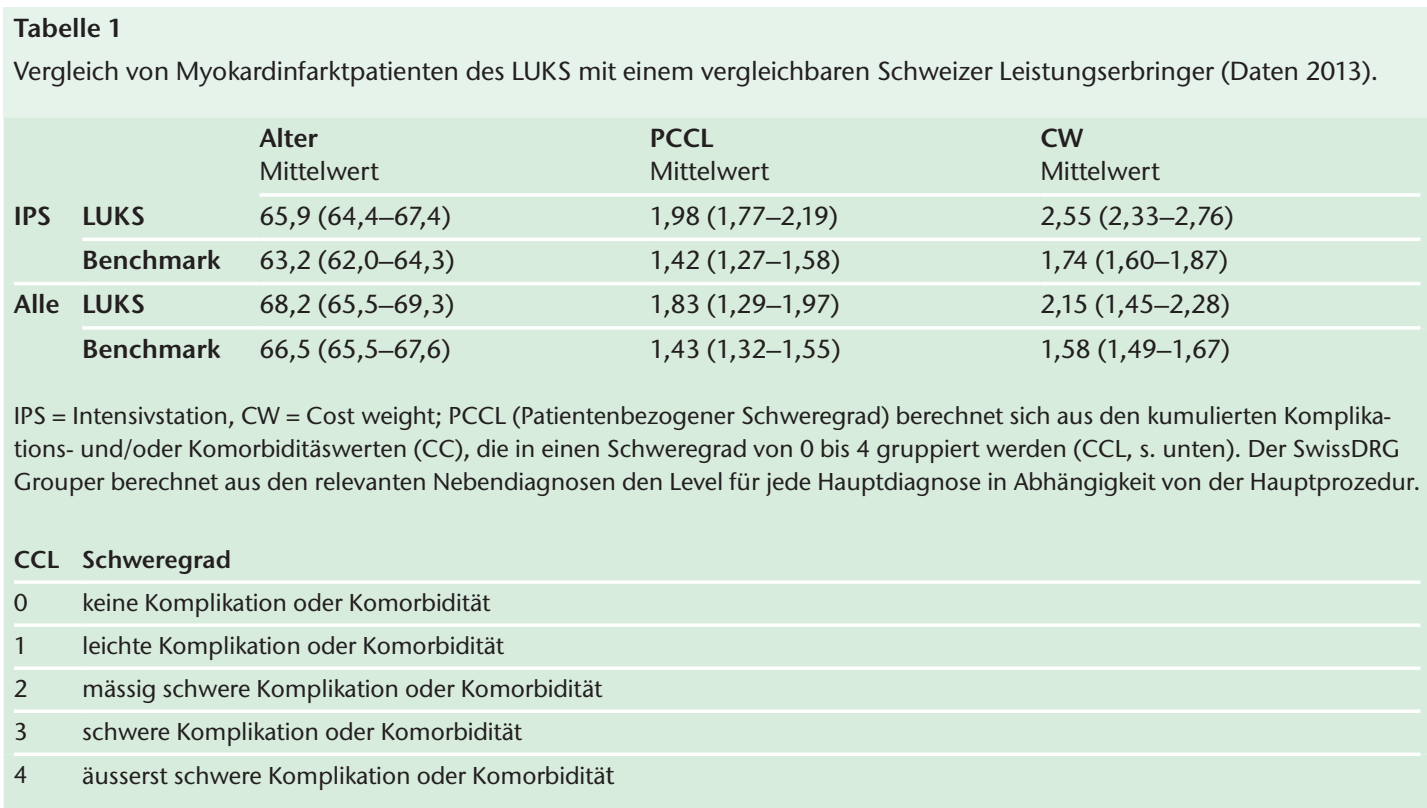


insgesamt also die Vermutung zulässig, dass die Unterschiede in der Infarktmortalitätsrate auf Grund unterschiedlicher Anwendungen der Diagnosekriterien, also der Ansteuerung von Zähler und Nenner erklärbar sind.

\section{Schlussfolgerungen}

Mortalitätsdaten auf der Basis von Routinedaten als Qualitätsindikatoren, die für Abrechnungszwecke generiert werden, wurden in den USA schon vor über 15 Jahren als untaugliches Instrument identifiziert [8]. Zudem variieren die Quoten abhängig vom angewandten Algorithmus [9]. Die eigentliche Versorgungsqualität ist lediglich für einen kleinen Teil der beobachteten Varianz der Mortalitätsraten zwischen Leistungserbringern verantwortlich. Mortalitätsraten sind nicht sensibel, um zwischen vermeidbaren und unvermeidbaren Todesfällen unterscheiden zu können [10]. Selbst das BAG verweist darauf, dass Morta- mente, um die Qualität in den unterschiedlichen Dimensionen der Indikationsstellung, der Strukturund Prozessqualität sowie der Ergebnisqualität zu managen. Dazu gehören Zertifizierungen (z. B. Chest Pain Unit), Peer-Reviews, Audits usw. Als akademisches Lehrspital legen wir Wert darauf, die Patienten in Studien zu behandeln. Dies führt zu Standardisierungen nach internationalen Standards und $\mathrm{zu}$ benchmarkfähigen Untersuchungsergebnissen, die dem Leistungserbringer helfen, unnötige Todesfälle $\mathrm{zu}$ vermeiden. Entscheidend ist die Identifikation von vermeidbaren Todesfällen. Die absolute Mortalitätsrate ist unwichtig, wenn keine Schwächen in der Struktur-, Prozess- oder Indikationsqualität aufdeckbar sind.

\section{Konklusionen}

Letalitätsdaten sind beliebte Qualitätsindikatoren. Sie sind jedoch kritisch zu hinterfragen bezüglich

\section{Die Versorgungsqualität ist lediglich für einen kleinen Teil der Varianz der Mortalitätsraten zwischen Leistungserbringern verantwortlich.}

litätsraten kein taugliches Instrument sind, um Aussagen zur Behandlungsqualität zu machen. Rein methodisch ist davon auszugehen, dass eine Risikoadjustierung alleine über Alter und Geschlecht ungenügend ist [11]. Sie erklärt lediglich rund 30\% der Rate. Mortalitätsraten ohne Vertrauensbereiche sind statistisch wertlos. Die LUKS-Letalitätsdaten im Abgleich mit Messungen der Erkrankungsschwere korrelieren jedoch signifikant mit der Mortalitätsrate.

Um beispielsweise bei Mortalitätsraten um 4\% einem Leistungserbringer eine exzessive Letalitätsrate nachzuweisen, braucht es zudem genügend grosse Fallzahlen.

Diese werden derzeit nicht erreicht. Bei prospektiv randomisierten Untersuchungen als wissenschaftlicher Goldstandard schneidet das Luzerner Kantonsspital mit den übrigen Zentrumsspitälern vergleichbar gut $a b$ und dies wurde auch in einer renommierten Zeitschrift entsprechend publiziert [12]. Das LUKS verwendet daher verschiedene Instru- der Validität von Zähler und Nenner. Deren Erzeugung müsste nachweislich vergleichbar entstehen. Qualitätsindikatoren müssen dazu dienen, vermeidbare Todesfälle zu identifizieren und so den Leistungserbringer befähigen, seine Indikations-, Prozess-, Struktur- und Outcomequalität zu verbessern. Eine Validierung von Zähler- und Nennerdaten fördert die Glaubwürdigkeit von Letalitätsdaten als sinnvolle Qualitätsindikatoren in einem ersten, aber auch unabdingbaren Schritt.

\section{Interaktiver Artikel}

Wollen Sie diesen Artikel kommentieren? Nutzen Sie dafür die Kommentarfunktion in der OnlineVersion oder sehen Sie nach, was Ihre Kolleginnen und Kollegen bereits geschrieben haben: www.saez.ch/aktuelle-ausgabe/interaktive-beitraege/ 


\section{Literatur}

1 Task Force on the management of STseamiotESoC, Steg PG, James SK, et al.

ESC Guidelines for the management of acute myocardial infarction in patients presenting with ST-segment elevation. European heart journal. 2012;33(20):2569-619.

2 Hamm CW, Bassand JP, Agewall S, et al.

ESC Guidelines for the management of acute coronary syndromes in patients presenting without persistent ST-segment elevation: The Task Force for the management of acute coronary syndromes (ACS) in patients presenting without persistent ST-segment elevation of the European Society of Cardiology (ESC). European heart journal. 2011;32(23):2999-3054.

3 Thygesen K, Alpert JS, Jaffe AS, et al. Third universal definition of myocardial infarction. European heart journal. 2012;33(20):2551-67.

4 Lim CC, van Gaal WJ, Testa L, et al. With the «universal definition», measurement of creatine kinase-myocardial band rather than troponin allows more accurate diagnosis of periprocedural necrosis and infarction after coronary intervention. Journal of the American College of Cardiology. 2011;57(6):653-61.

5 Parwani AS, Boldt LH, Huemer M, et al. Atrial fibrillation-induced cardiac troponin I release. International journal of cardiology. 2013;168(3):2734-7.

6 Landesberg G, Jaffe AS, Gilon D, et al. Troponin elevation in severe sepsis and septic shock: the role of left ventricular diastolic dysfunction and right ventricular dilatation. Critical care medicine. 2014;42(4):790-800.

7 Sasson C, Rogers MA, Dahl J, et al.

Predictors of survival from out-of-hospital cardiac arrest: a systematic review and meta-analysis. Circulation Cardiovascular quality and outcomes. 2010;3(1):63-81.

8 Iezzoni LI, Ash AS, Shwartz M, et al. Predicting who dies depends on how severity is measured: implications for evaluating patient outcomes. Annals of internal medicine. 1995;123(10):763-70.

9 Shahian DM, Wolf RE, Iezzoni LI, et al. Variability in the measurement of hospital-wide mortality rates. The NEJM. 2010;363(26):2530-9.

10 Lilford R, Pronovost P. Using hospital mortality rates to judge hospital performance: a bad idea that just won't go away. BMJ. 2010;340:c2016.

11 Kaufmann T, Lay V, Brach M, et al. Ein Vergleich von Registerdaten mit routinemässig erhobenen Datensätzen zur Messung der OutcomeQualität: Erfahrungen aus dem QuaZentral-Projekt aus der Schweiz. Gesundheitsökonomie \& Qualitätsmanagement. 2010;15(1):45-50.

12 Kaiser C, Galatius S, Erne P, et al. Drug-eluting versus bare-metal stents in large coronary arteries. NEJM. 2010;363(24):2310-9. 